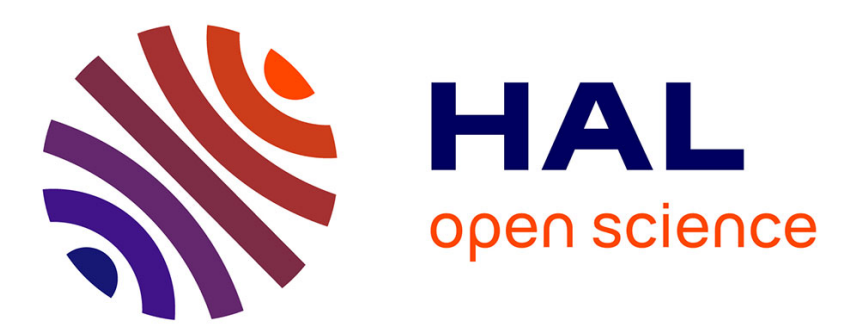

\title{
A use-dependent sodium current modification induced by type I pyrethroid insecticides in honeybee antennal olfactory receptor neurons.
}

Aklesso Kadala, Mercedes Charreton, Ingrid Jakob, Yves Le Conte, Claude Collet

\section{To cite this version:}

Aklesso Kadala, Mercedes Charreton, Ingrid Jakob, Yves Le Conte, Claude Collet. A use-dependent sodium current modification induced by type I pyrethroid insecticides in honeybee antennal olfactory receptor neurons.. NeuroToxicology, 2011, 32 (3), pp.320-30. 10.1016/j.neuro.2011.02.007 . hal00723020

\section{HAL Id: hal-00723020 https://u-bourgogne.hal.science/hal-00723020}

Submitted on 29 May 2020

HAL is a multi-disciplinary open access archive for the deposit and dissemination of scientific research documents, whether they are published or not. The documents may come from teaching and research institutions in France or abroad, or from public or private research centers.
L'archive ouverte pluridisciplinaire HAL, est destinée au dépôt et à la diffusion de documents scientifiques de niveau recherche, publiés ou non, émanant des établissements d'enseignement et de recherche français ou étrangers, des laboratoires publics ou privés.

\section{(이) $\$$}

Distributed under a Creative Commons Attribution - NonCommercial - NoDerivatives| 4.0 
Version définitive du manuscrit publié dans : NeuroToxicology, 2011, vol 32 : 320-330. DOI: 10.1016/j.neuro.2011.02.007/ Final version of the manuscript published in : NeuroToxicology, 2011, vol 32 : 320-330. DOI: 10.1016/j.neuro.2011.02.007

\title{
A use-dependent sodium current modification induced by type I pyrethroid insecticides
} in honeybee antennal olfactory receptor neurons

\author{
Aklesso Kadala ${ }^{1}$, Mercedes Charreton ${ }^{1}$, Ingrid Jakob ${ }^{2}$, Yves Le Conte ${ }^{1}$, Claude Collet ${ }^{{ }^{*}}$
}

Running title: Pyrethroids affect $\mathrm{I}_{\mathrm{Na}}$ in ORNs

Addresses:

${ }^{1}$ INRA, UMR406 Abeilles et Environnement, F-84000 Avignon, France

${ }^{2}$ CNRS, Centre Européen des Sciences du Goût, F-21000 Dijon, France

Author for correspondence:

Dr Claude COLLET

INRA, UMR406 Abeilles et Environnement, F-84000 Avignon, France

E-mail : claude.collet@avignon.inra.fr

Tel: (00 33) 432722649 ; Fax: (00 33) 432722602

Key words: Antennal sensilla, Olfactory receptor neurons, Sensory neuron, Electrophysiology, Patch-clamp, Insecticide, Permethrin, Tetramethrin, Ionic current, Bee health, Mode of action, Sodium channel, para, TipE, State-dependent modification 


\begin{abstract}
We studied the mode of action of type I pyrethroids on the voltage-dependent sodium current from honeybee olfactory receptor neurons (ORNs), which proper function in antenna is crucial for interindividual communication in this species. Under voltage-clamp, tetramethrin and permethrin induce a long lasting TTX-sensitive tail current upon repolarization, which is the hallmark of an abnormal prolongation of the open channel configuration. Permethrin and tetramethrin also slow down the sodium current fast inactivation. Tetramethrin and permethrin both bind to the closed state of the channel as suggested by the presence of an obvious tail current after the first single depolarization applied in the presence of either compounds. Moreover, at first sight, channel opening seems to promote tetramethrin and permethrin binding as evidenced by the progressive tail current summation along with trains of stimulations, tetramethrin being more potent at modifying channels than permethrin. However, a use-dependent increase in the sodium peak current along with stimulations suggests that the tail current accumulation could also be a consequence of progressively unmasked silent channels. Experiments with the sea anemone toxin ATX-II that suppresses sodium channels fast inactivation are consistent with the hypothesis that these silent channels are either in an inactivated state at rest, or that they normally inactivate before they open so that they do not participate to the control sodium current. In honeybee ORNs, three processes lead to a use-dependent pyrethroid-induced tail current accumulation: (i) a recruitment of silent channels that produces an increase in the peak sodium current, (ii) a slowing down of the sodium current inactivation produced by prolongation of channels opening and (iii) a typical deceleration in current deactivation. The use-dependent recruitment of silent sodium channels in honeybee ORNs makes pyrethroids more potent at modifying neuronal excitability.
\end{abstract}




\section{Introduction}

Within honeybees, social communication is mediated by odors and pheromones, that are detected by olfactory receptor neurons (ORN) localized in antennae. ORNs thus contribute to food source identification and interindividual recognition. Earlier studies showed that sublethal concentrations of type I pyrethroid insecticides (i) are present in hive waxes (Chauzat \& Faucon, 2007; Mullin et al., 2010) and (ii) can induce measurable behavioural modifications or impaired learning in honeybees (Cox \& Wilson, 1984; Taylor et al., 1987; Decourtye et al., 2005). While type I pyrethroids modes of action have been explored in heterologous systems (see below), comparatively few studies are available in a native neuronal environment and little is known about their modes of action in beneficial insects.

Voltage-activated sodium channels $\left(\mathrm{Na}_{\mathrm{V}}\right)$ underlie rapid neuronal electrical activity as they participate in action potential generation and propagation. Sodium channels are the primary targets for several pesticides classes (Bloomquist, 1996; Zlotkin, 1999; Soderlund et al., 2002; Dong, 2007). Among these pesticides, synthetic insecticides of the pyrethroid class are widely used against invertebrates known to be vectors of disease or to be parasites (e.g. mosquitos, acarians). Pyrethroids are also commonly used in crop protection against various insects considered as pests. These insecticides are known to affect the gating kinetics of neuronal sodium channels, causing repetitive discharges (with type I, non $\alpha$-cyano pyrethroids) or sustained neuronal membrane depolarization (with type II, that contain an $\alpha-$ cyano group) that in turn lead to symptoms of poisoning in animals, such as hyperexcitation (reviewed in Soderlund et al., 2002). In the fruit fly Drosophila melanogaster, the poreforming subunit of the $\mathrm{Na}_{\mathrm{V}}$ channel is encoded by the gene para. Orthologs for this gene have been identified in several invertebrate species including Anopheles gambiae, Aedes aegypti, Blattela germanica, Musca domestica, Heliothis virescens, Varroa destructor and Bombyx mori, but the full length clone cDNAs are available for four species only: Vssc1 from the house fly, DmNa $\mathrm{V}_{\mathrm{V}}$ from Drosophila melanogaster, BgNa $\mathrm{V}_{\mathrm{V}}$ from the cockroach and $\mathrm{VdNa}_{\mathrm{V}}$ from the varroa mite (for a review, see Dong, 2007). Recently, a para gene has been identified in the honeybee genome, sharing 97\% identity with the Drosophila gene (Whitfield et al., 2002; Honeybee Genome Sequencing Consortium, 2006).

Heterologous expression of the $\mathrm{Na}_{\mathrm{V}}$ alpha subunit in Xenopus oocyte allowed thorough characterization of $\mathrm{I}_{\mathrm{Na}}$ biophysical properties and pyrethroid sensitivity of invertebrate species, including Drosophila melanogaster, Blatella germanica and Varroa destructor, but has never been performed for the domestic honeybee (Feng et al., 1995; Warmke et al., 1997; Tan et al., 2002; Du et al., 2009). As a consequence, while toxic effects 
of pyrethroids on honeybee have been well described at the individual level (e.g. determination of lethal dose 50), their mode of action on voltage-gated sodium channels have been poorly documented in this beneficial insect as compared with data available on pests.

Since the voltage-dependent sodium current underlies finely tuned pheromones (and odors) coding in the honeybee peripheral olfactory nervous system, we aimed at characterizing the modes of action of two representative insecticides of the type I pyrethroid class, permethrin and tetramethrin on this current. We studied the traditional effects of these compounds and our experiments also explored another view of the pyrethroid-induced usedependent modification of sodium channels. To our knowledge, this is the first voltage-clamp characterization and quantification of pyrethroid-induced modifications in insect olfactory neurons. Part of this work was previously presented in abstract form (Kadala et al., 2010). 


\section{Materials and methods}

\subsection{Animals and cell culture}

Honeybee (Apis mellifera) worker prepupae were collected from hive combs in the research department experimental apiary. The development of the worker, which lasts 20-21 days, goes through five larval stages, a prepupal and a pupal stage to adult ecdysis. Drone and workerbee pupae are motionless and easily distinguishable from each other, on the basis of several anatomic features, including body size, aspect of the thorax seen beneath the moulted skin and number of segments that compose antennal flagellum i.e. 10 in workerbee and 11 in drone. Workerbee pupae were collected on day 11 before emergence, according to morphological changes of the cuticle (Jay, 1963). Antennal neurons were dissected and cultured following a protocol adapted from a procedure described in earlier studies (Gascuel et al., 1994; Laurent et al., 2002). In brief, prepupae were quickly dipped in 70\% ethanol for 5 seconds and rinsed with sterile-filtered distilled water. The cephalic part of the moulted larval skin that protects antennae was then opened in $\mathrm{Ca}^{2+}$ and $\mathrm{Mg}^{2+}$-free solution (in mM: 140 $\mathrm{NaCl}, 5 \mathrm{KCl}, 110$ sucrose, 10 HEPES, pH 7.2, $400 \mathrm{mOsm} / \mathrm{l}$, sterile-filtered) and the last nine segments from antennal flagella were dissected out and stored in a tube containing the same solution. Antennae fragments were enzymatically dissociated in a $\mathrm{Ca}^{2+}$ and $\mathrm{Mg}^{2+}$-free solution containing $0.04 \%$ trypsin $(\sim 10,000 \mathrm{UE} / \mathrm{mg})$ at $37^{\circ} \mathrm{C}$ for 12 minutes. After trypsin inactivation with an FBS-containing culture medium (see below) and centrifugation (1800 rpm, 3 minutes), the pellet was resuspended in culture medium. Culture medium was made of a commercial liquid Leibowitz 'L15' medium containing L-glutamine (Invitrogen), with added FBS (10\%), penicillin/streptomycin (1\%) and (in mM) 3.3 L-proline, 5.5 D-glucose, 75 sucrose (400 mOsm/l, pH 7.2 adjusted with $\mathrm{HCl}$ ). Dissociated tissue was passed 20 times through the tip of a $100 \mu \mathrm{l}$ pipette and the culture medium volume was adjusted so that one antennae was dispersed per $15 \mu$ l. Cells were plated on the central area $\left(\sim 1 \mathrm{~cm}^{2}\right)$ of poly-Llysine coated plastic Petri dishes, and cultured within a 'hanging drop column' (formed by a coverslip supported by two pieces of glass) as described earlier (Gascuel et al., 1994). Dishes were kept in an incubator $\left(29^{\circ} \mathrm{C}\right.$, high humidity) for up to 4 days.

\subsection{Scanning electron microscopy}

Adult honeybee (foragers) were anesthetized with $\mathrm{CO} 2$ or cold $\left(4^{\circ} \mathrm{C}, 30 \mathrm{~min}\right)$ and decapitated. Samples (heads or antennae) were placed on SEM stubs covered with double- 
sided tape and coated with gold (20 nm layer) in a Balzers sputter coater (model SCD004). Samples were observed with a Phillips-FEI XL30 scanning electron microscope at $10 \mathrm{kV}$ (magnifications 70-10,000×). Experiments were performed in the Pathologie Végétale Department in Avignon.

\subsection{Electrophysiology.}

Neurons were used between 2 and 4 days of in vitro cell culture. A patch-clamp amplifier (RK400, Bio-Logic, Claix, France) was used in the whole-cell configuration to measure membrane currents. Voltage pulse generation and data acquisition were done using WinWCP software (John Dempster, Strathclyde University, UK) driving an A/D, D/A converter (PCI-6014 board, National instruments Corp. Austin, TX, USA). Patch-clamp pipettes were pulled from borosilicate glass capillaries on a vertical pipette puller (P30, Sutter Instrument Co, Novato, AS, USA). Sylgard-coated electrodes were used to minimize the residual pipette capacitance that was compensated analogically. The resistance of microelectrodes filled with internal solution (see Solutions) ranged between 6 and $10 \mathrm{M} \Omega$ in standard extracellular solutions. Microelectrode offset potential was nulled prior to seal formation and residual microelectrode capacitance was zeroed with a fast analog compensation circuit available on the amplifier, after gigaseal formation, before membrane patch rupture. Before reaching whole-cell configuration, the resting holding potential was established at $-80 \mathrm{mV}$. Capacitance and series resistances were maximally compensated for (60-80\%). Passive leak currents and residual linear capacitative currents were subtracted as described earlier (Collet, 2009) or using a P/4 protocol where each test pulse was preceded (at least 1 second before) by a series of 10 pulses of amplitude of one fourth in the opposite direction (this subtraction induced a decrease in the signal to noise ratio with slightly noisier traces, but more accurate measurement of current amplitudes). All experiments were performed at room temperature $\left(20-22^{\circ} \mathrm{C}\right)$.

\subsection{Solutions for electrophysiology}

Standard extracellular solution contained (in mM) : $120 \mathrm{NaCl}, 20$ TEA-Cl, $2 \mathrm{MgCl}_{2}, 2$ $\mathrm{BaCl}_{2}, 0.1 \mathrm{CdCl}_{2}, 1$ 4-aminopyridine, $10 \mathrm{HEPES}, 90$ sucrose, adjusted to $\mathrm{pH} 7.2$ with $\mathrm{HCl}$ and to $400 \mathrm{mOsm} / \mathrm{l}$ with sucrose. During experiments with pyrethroids, DMSO was also added to this solution $(0.1 \%$, matching with the concentration present in the pyrethroid-containing solution). Intracellular (pipette) solution contained (in mM): $135 \mathrm{CsCl}, 5 \mathrm{NaCl}, 1 \mathrm{MgCl}_{2}, 1$ $\mathrm{CaCl}_{2}$, 10 EGTA, 10 HEPES, 90 sucrose, pH 7.2 (380 mOsm/l). When appropriate, cells were exposed to various experimental solutions by means of a computer-operated gravity driven 
perfusion system (perfusion rate $0.3 \mathrm{ml} . \mathrm{min}^{-1}$ ) described earlier (Collet \& Belzunces, 2007). Each Petri dish was discarded after perfusion (only one neuron was recorded per dish) and the tubing system and reference electrode were thoroughly rinsed with running 70\% EtOH and distilled water after each series of experiments. Tetramethrin (mixture of isomers, $20 \%$ cis and $80 \%$ trans) and permethrin (mixture of isomers, $28 \%$ cis and $72 \%$ trans) stock solutions (10 $\mathrm{mM})$ were prepared daily in DMSO and dissolved in the perfusion solution (DMSO concentration never exceeded $0.1 \%$ ). Tetrodotoxin was used at a final concentration of $1 \mu \mathrm{M}$ in the external solution. All compounds were purchased from Sigma-Aldrich. The sea anemome toxin ATX-II (provided by Dr S. Diochot, IPMC, Valbonne, France) was directly dissolved in the extracellular saline solution and used at a final concentration of $20 \mathrm{nM}$.

\subsection{Data analyses}

Patch-clamp data were analyzed with Origin Pro. Least-square fits were performed using a Levenberg-Marquardt algorithm routine included in Origin. Values are given as means \pm standard errors of the means (S.E.M.). Individual curves of the sodium current voltage dependence were fitted with Eqn 1: $\left.I(V)=G_{\max }\left(V-V_{\text {rev }}\right) /\left\{1+\exp \left[V_{0.5}-V\right) / k\right]\right\}$, where $\mathrm{I}(\mathrm{V})$ is the peak density of the current for a depolarization to a membrane potential $V, G_{\max }$ is the maximum conductance, $\mathrm{V}_{\text {rev }}$ is the apparent reversal potential, $\mathrm{V}_{0.5}$ is the half activation voltage and $\mathrm{k}$ is a steepness factor. Steady-state activation and inactivation experimental data were fitted with Boltzman equations. Eqn 2 and 3 were used: $m_{\infty}=1 /\left\{1+\exp \left[\left(V_{m}-V\right) / K_{m}\right]\right\}$ and $h_{\infty}=1 /\left\{1+\exp \left[\left(V-V_{h}\right) / K_{h}\right]\right\}$, where $V_{m}$ and $V_{h}$ are the potential for half activation and inactivation respectively and $\mathrm{K}_{\mathrm{m}}$ and $\mathrm{K}_{\mathrm{h}}$ are slope parameters. The percentage of channels modified by pyrethroids was calculated using Eqn $4: \mathrm{M}=\left\{\left[\mathrm{I}_{\text {taii }} /\left(\mathrm{E}_{\mathrm{h}}-\mathrm{E}_{\mathrm{rev}}\right)\right] /\left[\mathrm{I}_{\mathrm{Na}} /\left(\mathrm{E}_{\mathrm{t}}-\mathrm{E}_{\mathrm{Na}}\right)\right]\right\} \mathrm{x}$ 100 , where $I_{t a i l}$ is the maximal tail current amplitude, $E_{h}$ is the potential to which the membrane is repolarized, $E_{\text {rev }}$ is the reversal potential for sodium current determined from the current voltage curve, $\mathrm{I}_{\mathrm{Na}}$ is the amplitude of the current during depolarization and $\mathrm{E}_{\mathrm{t}}$ is the membrane potential reached during the test pulse (Tatebayashi \& Narahashi, 1994). I tail $_{\text {was }}$ measured right after the test pulse by extrapolation of a monoexponential equation fitted to the tail current decay. The concentration-response data were fitted with the Hill equation (Eqn 5), $\mathrm{M}=\mathrm{M}_{\max } /\left[1+\left(\mathrm{K}_{\mathrm{d}} /[\mathrm{Pyr}]\right)^{\mathrm{n}}\right]$, where [Pyr] is the concentration of pyrethroid, $\mathrm{K}_{\mathrm{d}}$ is the concentration that produced the half-maximal effect, $n$ is the Hill coefficient, and $M_{\max }$ is the maximal percentage of modified sodium channels (Tatebayashi \& Narahashi, 1994). Statistical analysis was performed using a paired Student's t-test assuming significance for $\mathrm{P}<0.05$. 


\section{Results}

\subsection{Olfactory receptor neurons from honeybee antenna}

In honeybee, ORNs are located in sensillae covering antennae and are extremely important for chemical communication in the hive and proper foraging activity (Fig. 1A and B). Scanning electron microscopic observations allow morphological identification of various sensillae types (Fig. 1C and D). Sensillae placodea, that appear as oval cuticular plates (Fig. $1 \mathrm{E})$ are the main olfactory antennal structures in honeybee and are innervated by 35 to 50 ORNs (Esslen \& Kaissling, 1976). ORNs were obtained from prepupae antennae (Fig. 1F and G). We recorded electrophysiological signals from ORNs that are the most abundant cell population and are easily distinguishable by their size, shape and because they are often gathered in clusters in cell culture (Fig. 1H).

\subsection{Steady-state activation and inactivation curves for $\mathbf{I}_{N a}$}

Several biophysical properties of the ORN voltage-dependent sodium current were explored. First, current-voltage (I/V) relationships were obtained from individual neurons. Fig. 2A shows the mean normalized maximal amplitude of $\mathrm{I}_{\mathrm{Na}}$ between -70 and $+70 \mathrm{mV}$ obtained from twenty-two ORNs. Currents were readily voltage-clamped as demonstrated by a shallow I/V curve near activation threshold potentials. Threshold for activation was between -50 and $-40 \mathrm{mV}$ and $\mathrm{I}_{\mathrm{Na}}$ maximal current was obtained between -10 and $0 \mathrm{mV}$. The apparent reversal potential was $+71 \mathrm{mV}$, close to the theoretical sodium equilibrium potential calculated according to the Nernst equation in our experimental conditions (83 mV). On average, $I_{\mathrm{Na}}$ had a maximal amplitude of $87 \pm 10$ pA (n=22). A two-step pulse series of experiments (Fig. 2Ba) was performed to measure both activated (first pulse) and inactivated (second pulse) fractions of $\mathrm{I}_{\mathrm{Na}}$ (Fig. 2Bb). A steady state activation curve ( $\mathrm{m}_{\infty}$, grey circles) and a steady state inactivation curve ( $\mathrm{h}_{\infty}$, black circles) were averaged from five ORNs (Fig. 2Bc). Curves were fitted to the data according to the Boltzmann equations (Eqn 2 and 3) with $\mathrm{V}_{\mathrm{m}}$ and $\mathrm{V}_{\mathrm{h}}$ (the potential for half activation and inactivation, respectively) of -21.1 and -42.1 $\mathrm{mV}$ and $\mathrm{K}_{\mathrm{m}}$ et $\mathrm{K}_{\mathrm{h}}$ (slope factors) of $6.9 \mathrm{mV}$ and $6.2 \mathrm{mV}$.

\subsection{Properties of the pyrethroid-induced sodium tail currents in honeybee ORN}

No tail current developed after the depolarizing pulse in control conditions (Fig. 3A, left trace). In contrast, when ORNs were superfused with permethrin (10 $\mu \mathrm{M}, 3 \mathrm{~min})$, a pronounced tail current appeared after membrane repolarization (Fig. 3A, middle trace). The tail current was already present after the very first pulse applied in permethrin. Tetrodotoxin $(1 \mu \mathrm{M})$ completely blocks both $\mathrm{I}_{\mathrm{Na}}$ (during the pulse) and the tail current after the pulse (right trace). TTX blocking effect on control $\mathrm{I}_{\mathrm{Na}}$ was observed in all ORNs exposed $(n=7)$. The TTX 
blocking effect of the permethrin-induced tail current was seen in all ORNs exposed to both compounds $(\mathrm{n}=3)$. To further characterize the permethrin effect, a protocol aimed at exploring the reversal potential of the permethrin-induced tail current was used (Fig. 3B, pulse protocol). The maximal amplitude of the tail current occurring after a 3 ms pulse to $-10 \mathrm{mV}$ was measured for membrane potentials ranging from -80 to $+60 \mathrm{mV}$ (Fig. 3B, lower series of traces from one individual neuron). Mean maximal amplitude of the tail current was obtained in two ORNs within this range of membrane potentials (Fig. 3C, filled circles). A linear function adjusted to these data gave an apparent reversal potential of $+42 \mathrm{mV}$ for this tail current. No inward tail current could be recorded in three ORNs with this protocol in the absence of permethrin (empty circles), with an even slightly outward I/V curve in control conditions for the most positive potentials. When subtracting the control I/V curve from the I/V curve in presence of permethrin, the reversal potential was shifted toward a more positive value, closer to the theoretical sodium equilibrium potential (not shown).

As for permethrin, a tetramethrin-induced tail current was present after the very first depolarization pulse applied after equilibration in the pyrethroid-containing solution. Interestingly, in our experimental conditions, the pyrethroid-induced sodium tail current was fully reversible with tetramethrin but not with permethrin. Hence, as illustrated in Figure 4A, a prominent tail current appeared in the presence of tetramethrin $10 \mu \mathrm{M}(7 \mathrm{~min}, 10 \mu \mathrm{M})$ and this effect was fully reversed after ten minutes of washout with a control solution (the complete reversibility was demonstrated in three other neurons). A tail current was also observed in the presence of permethrin $(9 \mathrm{~min}, 10 \mu \mathrm{M})$, but this effect was not completely reversed after 12 minutes of washout with control solution (Fig. 4B).

Tetramethrin and permethrin (10 $\mu \mathrm{M}, 3$ min exposure), not only induced a tail current after repolarization from a single short pulse, but the $\mathrm{I}_{\mathrm{Na}}$ amplitude, measured at the end of the depolarizing pulse, also increased (Fig. 5A and B, filled circle). Conversely, no effect was found for the peak of the sodium current during depolarization in the presence of either tetramethrin or permethrin (Fig. 5A and B, empty circle). Mean amplitudes at the peak (empty bars) and at the end of the pulse (filled bars) were obtained from five ORNs in tetramethrin 10 $\mu \mathrm{M}$ (Fig. 5C, left) and eleven ORNs in permethrin $10 \mu \mathrm{M}$ (Fig. 5C, right). No significant pyrethroid-induced effect was observed for the peak $(\mathrm{P}=0.2$ in tetramethrin and $\mathrm{P}=0.4$ in permethrin), but there was a statistically significant increase for the late part of the current, revealing a slower $\mathrm{I}_{\mathrm{Na}}$ inactivation $(\mathrm{P}=0.03$ in tetramethrin and $\mathrm{P}=0.007$ in permethrin).

Figure 6A shows the superimposed traces of $\mathrm{I}_{\mathrm{Na}}$ in response to a single $3 \mathrm{~ms}$ long depolarization and the resulting tail currents recorded in three individual ORNs exposed to 
tetramethrin $10 \mu \mathrm{M}$. The time course of $\mathrm{I}_{\mathrm{Na}}$ tail currents was estimated by fitting a monoexponential function to the decay of this tail current for each individual ORN. On average, mean decay time constant was $117 \pm 37 \mathrm{~ms}$ (range 57 to $183 \mathrm{~ms}, \mathrm{n}=3$ ). The amplitude of the tetramethrin-induced tail current was measured at the end of recordings (130 ms after the end of the pulse, arrow) for each ORN and compared to the maximal tail amplitude right after repolarization. On average, after this delay, the residual tail current amplitude was still $30 \pm 12 \%$ of its maximal value (Fig. 6B, n=3). Figure 6D shows the superimposed traces of $\mathrm{I}_{\mathrm{Na}}$ in response to a single $3 \mathrm{~ms}$ long depolarization and the resulting tail currents recorded in eleven individual ORNs exposed to permethrin $10 \mu \mathrm{M}$. Since the permethrin-induced tail current decay was sometimes much slower than in tetramethrin, no attempt was made to systematically fit its decay. The mean residual tail current amplitude was $51 \pm 6 \%$ at the end of recordings ( $n=11$, Fig. $6 \mathrm{E}$ ), indicating that on average, permethrin produces slower tail currents than tetramethrin.

\subsection{Percentage of modified sodium channels in bee ORN exposed to pyrethroids}

The tail current amplitude right after repolarization was used to calculate an estimation of the percentage of modified channels in the presence of tetramethrin and permethrin $10 \mu \mathrm{M}$ (according to Eqn 4). On average, $22 \pm 7 \%$ of the channels were modified in the presence of tetramethrin (Fig. 6C, $n=3$ ). In the presence of permethrin, $20 \pm 4 \%$ of the channels were modified (Fig. 6F, n=11). Dose-response curves were obtained in ORNs exposed to increasing concentrations of permethrin. An example of tail currents obtained after a single 3 ms depolarization in one ORN in the presence of permethrin at $0.1,1,5$ and $10 \mu \mathrm{M}$ is given in Figure 7 (inset). In a total of nine ORNs, tail currents were measured in control and in the presence of four or five different permethrin concentrations applied sequentially ( 0.1 to 50 $\mu \mathrm{M})$ and the percentages of modified channels were calculated. Figure 7 shows the mean percentage of modified channels from dose-responses made in individual ORNs. Averaged percentages were made for each concentration with the available number of values (three for $0.1 \mu \mathrm{M}$, nine for $1 \mu \mathrm{M}$, nine for $3 \mu \mathrm{M}$, nine for $10 \mu \mathrm{M}$ and five for $50 \mu \mathrm{M}$ ). In this series of experiments, where ORNs were subjected to several permethrin concentrations, the mean percentages of modified channels were: $0.6 \pm 0.3 \%(0.1 \mu \mathrm{M}), 4.4 \pm 0.5 \%(1 \mu \mathrm{M}), 22.8 \pm 3.7$ $\%(5 \mu \mathrm{M}), 25.6 \pm 3.8 \%(10 \mu \mathrm{M}), 34 \pm 8.5 \%(50 \mu \mathrm{M})$. Consistent with the previous series of experiments (single concentration, Fig. 6F), the mean percentage of modified channels calculated for $10 \mu \mathrm{M}$ permethrin in this multiple-concentrations series of experiments was not significantly different from the mean value obtained in the single concentration experiments. A Hill equation (Eqn 5) was adjusted to the data, with a $\mathrm{K}_{\mathrm{d}}$ of $3.5 \mu \mathrm{M}$. 


\subsection{Use-dependency of the pyrethroid effect on ORNs}

In experiments presented above, the modified channels fraction was assessed after a single short pulse. Trains of short depolarizing pulses should mimic a train of action potentials with regard to activation of sodium channels. In order to explore the consequence of a stimulation arising while the tail current has not completed its decay, we next investigated current responses during trains of short pulses. We determined the optimal stimulation frequency for such a protocol by characterizing the $\mathrm{I}_{\mathrm{Na}}$ use-dependent decrease (Roy \& Narahashi, 1992; Blair \& Bean, 2003) and recovery from fast inactivation. First, a two-pulses protocol was used in order to evaluate recovery from inactivation (Fig. 8A). The inset shows two examples of superimposed current series obtained during two consecutive depolarizing pulses from -80 to $-10 \mathrm{mV}$ (3 ms and $5 \mathrm{~ms}$ long pulse for upper and lower trace, respectively). Interpulse time was ranging from 1 to $13 \mathrm{~ms}$ with an increment of $1 \mathrm{~ms}$ (series 1: 3 ms pulse, empty symbols) or 3 to $21 \mathrm{~ms}$ with an increment of $5 \mathrm{~ms}$ (series 2: $5 \mathrm{~ms}$ pulse, filled symbols). The recovered fraction of $I_{\mathrm{Na}}\left(I_{2}\right)$ was normalized to $I_{1}$ and plotted on the graph as a function of recovery time. No attempt was made to mathematically adjust an exponential function on these experimental scattered points (a monoexponential with a time constant of 4.8 ms was fitted by eye). $\mathrm{I}_{\mathrm{Na}}$ recovery tended to be almost complete for the longest interpulse duration, $28 \mathrm{~ms}$. Cumulated use-dependent reduction of the sodium current peak during repetitive depolarizations was explored in control conditions since such protocol was later on used to assess $\mathrm{I}_{\mathrm{Na}}$ properties in the presence of insecticide (Fig. 8B). Trains of short depolarizations ( $3 \mathrm{~ms}$ to $-10 \mathrm{mV}$ ) were applied at frequency of 35 or $125 \mathrm{~Hz}$, that is one pulse every $28 \mathrm{~ms}$ (upper trace) or every $8 \mathrm{~ms}$ (lower trace), respectively. Averaged normalized amplitudes ( $\mathrm{I} / \mathrm{I}_{\max }$, were $\mathrm{I}_{\max }$ is the amplitude of the current during the first pulse) along with $35 \mathrm{~Hz}$ - or $125 \mathrm{~Hz}$ - train protocols were obtained for 10 and 5 ORNs, respectively (lower panel). Whereas $35 \mathrm{~Hz}$ train leads to a weak and shallow sodium current usedependent reduction, a steeper reduction was obtained for the $125 \mathrm{~Hz}$ train, with a pronounced effect seen between the first and the second pulses. A $35 \mathrm{~Hz}$ protocol was thus chosen to assess pyrethroid effects during a repetitive stimulation protocol.

A cumulative effect was observed when trains of short stimulations were applied to pyrethroid-exposed ORNs (Fig. 9). In control conditions (no pyrethroid), no sodium tail current can be detected after either a single 3ms pulse or a train of ten 3-ms pulse at $35 \mathrm{~Hz}$ (see Fig. 8B). Conversely, in the presence of tetramethrin (Fig 9A, top trace) or permethrin 10 $\mu \mathrm{M}$ (Fig 9A, bottom trace), a tail current is not only present after the end of the first 3ms pulse, but also increases in amplitude along with consecutive pulses. A cumulative effect is 
thus observed with a train of short pulses at $35 \mathrm{~Hz}$, with a summation of successive tail currents following each 3ms pulse. In Fig. 9B (left panel), the tail current amplitude increase is plotted for the two neurons shown in Fig. 9A bathed in tetramethrin (white dots) and permethrin (black dots), respectively (superimposed curves were drawn by eye). This cumulative effect was observed in every ORN bathed with tetramethrin $(n=4)$ or permethrin $(n=13)$. Average tail current increases are presented in Fig. 9B (right panel) for tetramethrin (white dots) and permethrin (black dots), respectively. The time constant of tail current accumulation was obtained by fitting a monoexponential curve to the averaged data and was $49 \mathrm{~ms}$ and $82 \mathrm{~ms}$ for tetramethrin- and permethrin-exposed neurons, respectively. Interestingly enough, while the control peak current only slightly decreased along with successive stimulations at $35 \mathrm{~Hz}$ (Fig 8B and 9C top trace), a peak current increase could be seen in a majority of pyrethroid-exposed ORNs (three out of four neurons in tetramethrin and ten out of thirteen neurons in permethrin). An illustration of this phenomenon is given in Fig 9C for one ORN before (top trace) and after exposure to permethrin (bottom trace). Whereas in control, the peak current slightly decreased along with stimulations, the peak gradually increased when the neuron was exposed to permethrin (successive current peaks are indicated by arrows). From one neuron to another, this current increase was observed for the three to five first stimulations. This increase in the peak current suggests that repeated stimulations progressively recruit a population of channels that were silent during the first step (noncontributing channels), either in the closed or inactivated state.

Existence of inactivated channels is not obvious when looking at the steady-state activation curve, that suggests that all channels are already in an activatable status at our holding potential $(-80 \mathrm{mV}$, Fig 2Bc). However, to explore the possibility that such a population of inactivated channels exist, ATX-II, the sea anemone toxin well known to relieve sodium channels from the fast inactivation process was used. On average, in four neurons, in the presence of ATX-II (20 nM), a long lasting sodium current appeared with inactivation slowed down by 190 fold as compared with the fast inactivating current in control (Fig 9D, top trace, $100 \mathrm{~ms}$ pulse to $-10 \mathrm{mV}$ ). The ATX-II- induced inactivation blockade was accompanied by an increase in the current peak amplitude. The mean peak of the sodium current was increased $\sim 1.5$ fold $(n=4)$ suggesting that in control conditions, at Vmax, a fraction of channels inactivate before they open and do not contribute to the sodium current. A train of ten depolarizing pulses at $35 \mathrm{~Hz}$ could not recruit more peak current (Fig 9D, bottom trace). As expected, ATX-II had no effect on the deactivation process, as evidenced by the lack of tail current. In the presence of ATX-II, the permethrin-induced tail 
current after a single pulse (Fig 9E, right trace) reaches a higher amplitude than the one reached after ten pulses in the absence of toxin (Fig 9E, left trace). On average, with ATX-II, the amplitude after a single pulse is 1.6 fold bigger $(n=3)$. Thus, in permethrin, ten pulses do not appear sufficient to unmask all silent channels, as suggested in Fig 9B, since the tail current has apparently not reached its maximum yet. Furthermore, experiments with ATX-II suggest that the speed of tail current accumulation (that is slightly different for the two pyrethroids tested here) might be mainly limited not only by the kinetics of tail current decay (compare the tail current decay in tetramethrin and permethrin in Fig 9A), but also by the inactivation of the pyrethroid-modified sodium current. The use-dependent tail current accumulation phenomenon thus results from equilibrium in between (i) the deactivation process, (ii) the fast inactivation process and (iii) the use-dependent reduction of $\mathrm{I}_{\mathrm{Na}}$ peak. The increase in the peak current along with successive stimulations in the presence of pyrethroid, and the recruitment of inactivated channels by ATX-II suggest that in honeybee ORNs, a pyrethroid-induced recruitment of fast inactivating channels is at least partly responsible for the use-dependent increase of the tail current. 


\section{Discussion}

Our examinations of the voltage-dependent sodium current in honeybee antennal olfactory receptor neurons suggest a strong sensitivity of these neurons to type I pyrethroid insecticides. To date, several major findings about pyrethroids modes of action have been obtained in experimental systems employing cloned insect channels heterologously expressed in Xenopus oocytes. By contrast, studies involving insect sodium channels kept in their native intracellular neuronal environment are not abundant (Lee et al., 1999; Martin et al., 2000; Park et al., 2000). As described here, honeybee ORNs provide a convenient system to study pyrethroids modes of action on sodium channels in their intracellular environment. Moreover, owing to their peripheral localization in antenna, ORNs could be chronically exposed to these insecticides. Interestingly, sublethal effects of type I pyrethroids have been reported on honeybee (Cox \& Wilson, 1984; Taylor et al., 1987), and mainly interpreted as central nervous system perturbations, while the effects on antennae were so far not explored in spite of the crucial importance of this organ in honeybee.

Permethrin and tetramethrin induce a TTX-sensitive fast activating- and slowly decaying- sodium tail current upon repolarization under voltage-clamp conditions. The slow decay of the tail current is commonly interpreted as the slowing down of sodium channels activation gate $(\mathrm{m})$ closure. One fifth of the channels appear modified by concentrations of 10 $\mu \mathrm{M}$ permethrin or tetramethrin after a single short depolarizing pulse at $\mathrm{V}_{\text {max }}$. Whereas a pronounced tail current was induced by tetramethrin or permethrin after membrane repolarization in honeybee ORNs, the peak amplitude of the sodium current during a single depolarization was not significantly affected by these two pyrethroids. However, fast inactivation of $\mathrm{I}_{\mathrm{Na}}$ was apparently slowed down during the pulse since the current amplitude at the end of the pulse was significantly larger (Fig.5). Permethrin and tetramethrin-induced sodium current modification during a step depolarization in honeybee ORNs was consistent with earlier works on Heliothis virescens central neurons (Park et al., 2000), oocytes expressing Drosophila melanogaster sodium channels (Warmke et al., 1997) and TTX-S endowed rat DRG neurons (Tatebayashi \& Narahashi, 1994; Song et al., 1996) where $\mathrm{I}_{\mathrm{Na}}$ amplitude was significantly increased at the end of the depolarizing pulse but apparently not at the peak current. Interestingly, in a non-insect invertebrate (squid giant axon), tetramethrin decreased the sodium current peak by 2 to $8 \%$, (Lund \& Narahashi, 1981a). For other type I pyrethroids (e.g. allethrin), more important effects have been described, a decrease in temperature intensifying the blocking action (Narahashi, 1971). These discrepancies could be due to species, preparations, compounds or experimental temperatures differences. 
Tetramethrin -induced tail currents in cultured honeybee ORN appear fully reversible, at least at the time scale considered and in our experimental conditions (in the presence of DMSO 0.1\%). Permethrin effects are hardly reversible in the same experimental conditions. The reversibility after washout of tetramethrin has been well documented before, both in vertebrate and invertebrate preparations. Recovery was usually found much faster in rat cerebellar Purkinje neurons or rat DRG neurons than in crayfish or squid giant axons (Lund \& Narahashi, 1981b; 1982; Song et al., 1996; Song \& Narahashi, 1996). Here, we found that reversibility of the tetramethrin effect was as fast as in studies on vertebrate neurons. In a previous study, we have also shown that allethrin induced an only partially reversible action potential inhibition in honeybee skeletal muscle fibers, mediated by $\mathrm{Ca}^{2+}$ current blockade (Collet \& Belzunces, 2007; Collet, 2009). Narahashi and colleagues studied in great detail the reversibility of allethrin-induced modifications in a system where effects are only governed by partitioning into the neuronal membranes and binding of sodium channels. It is worthy to mention that in these patch-clamp experiments, tail currents induced by allethrin (another type I pyrethroid) in cultured rat DRG neurons are reversible with much faster kinetics when DMSO $0.1 \%$ is present ( $\mathrm{t}=144 \mathrm{~s}$ vs tens of minutes in the absence of DMSO, Ginsburg \& Narahashi, 1999), a concentration that we routinely used in our control solutions. Time course of reversibility appears to depend on the compound lipophilic characteristics, with slower time courses being obtained with type II pyrethroids (Tabarean \& Narahashi, 1998).

Although quantification of free pyrethroid insecticide concentrations in close proximity with honeybee ORN in situ has never been performed, recent studies demonstrated the presence of type I pyrethroids not only in waxes and pollens, but also in bees body (Chauzat \& Faucon, 2007; Mullin et al., 2010). Our dose-response experiments suggest that permethrin is at least as potent on honeybee sodium current as on sodium currents from Heliothis Virescens, Musca domestica and Drosophila melanogaster, with an $\mathrm{EC}_{50}$ in the micromolar range (Lee et al., 1999; Zhao et al., 2000; SupYoon et al., 2008). Elevated pyrethroid concentrations are employed in voltage-clamp studies to characterize the effects of pyrethroids on sodium currents in detail and to assess the relative sensitivity of channels to pyrethroid modification, but several orders of magnitudes lower pyrethroids concentrations are known to disrupt normal electrical signaling in intact neurons. In cultured honeybee ORNs, less than $1 \%$ of the channels are modified by the lowest permethrin concentration tested $(0.1 \mu \mathrm{M})$ after a single depolarization, a percentage that induces abnormal repetitive afterdischarges in mammalian central neurons (Song \& Narahashi, 1996). 
In honeybee ORNs, our results show that the tetramethrin and permethrin-induced tail currents easily undergo accumulation upon repetitive stimulations, because the tail currents can have decay time constants in the range of several tens or hundreds of milliseconds. These sustained tail currents recall prolonged channel opening underlying in vivo deleterious neuronal hyperexcitability described for type I pyrethroids in other organisms (reviewed for instance in Soderlund et al., 2002). The sodium tail current accumulation phenomenon has been described for pyrethroids and other neurotoxic compounds of the veratrum plant alkaloid family and tentatively explained as a use-dependent modification of the channel population with the progressive recruitment upon channel opening (Barnes \& Hille, 1988; Smith et al., 1998; Vais et al., 2000; Tabarean \& Narahashi, 2001). However this use-dependence mechanism is far from being fully elucidated (Soderlund, 2010). In our case, tail current summation along with repetitive stimulations could be the result of progressive recruitment of silent channels along with consecutive stimulations, as evidenced by an increase in the sodium current peak. The detection of this peak current increase was permitted by a preliminary determination of the peak current use-dependent reduction (Roy \& Narahashi, 1992; Blair \& Bean, 2003) and the characterization of its recovery from fast inactivation in control conditions. At least two studies made on heterologously expressed insect para/TipE have shown previously that many insect sodium channels do not normally open during a strong depolarization (Warmke et al., 1997; Vais et al., 2000). These authors described a twofold increase in $\mathrm{G}_{\mathrm{Na} \text {,max }}$ (maximal sodium conductance) with the toxin ATX-II ( $>100 \mathrm{nM}$ ). A pool of silent channels also exists in honeybee ORNs, as demonstrated by an ATX-IIpromoted increase in the sodium current at Vmax. In fact ATX-II modifies the availability of para/TipE channels by inhibiting not only channel inactivation from the open state, but also from the closed state (Warmke et al., 1997; Vais et al., 2000). The pyrethroid-induced usedependent increase in the sodium current peak observed in our study could be the result of a use-dependent recruitment of this pool of non-available (silent) channels, but further experiments would be necessary to investigate this hypothesis. In addition, accumulation of persistently open pyrethroid-modified channels upon repeated depolarizations (Tabarean \& Narahashi, 2001) or recruitment of channels after their opening (Smith et al., 1998; Vais et al., 2000; Vais et al., 2003) are likely involved in the use-dependent tail current summation that we describe in honeybee ORN. From our experiments, it is clear that a single depolarization is capable of promoting a tail current in honeybee ORNs in the presence of tetramethrin or permethrin. This result has been interpreted in other preparations as a capacity for type I pyrethroids such as cismethrin (Smith et al., 1997; Smith et al., 1998) and 
permethrin (Warmke et al., 1997; SupYoon et al., 2008) to bind closed sodium channels. However, it takes a series of repeated ORNs depolarizations to eventually enhance recruitment of all channels available for the modification. Interestingly, in Xenopus oocytes heterologously expressing Musca domestica sodium channels, cismethrin modifies all channels in their closed state, as evidenced by a lack of use-dependence, suggesting the fact that multiple stimulations cannot recruit more modified channels than a single depolarization (Smith et al., 1998). In our case, tetramethrin tends to behave as an intermediate compound in between cismethrin and permethrin, since a single depolarization appears to recruit the majority of modifiable channels, but two or three additional stimulations are needed to reach the maximal tail current. The channel use-dependency in the presence of various pyrethroids has been correlated with the speed of the tail current decay and the speed of channel activation : the faster the tail current decays, the least use-dependency (Choi \& Soderlund, 2006). In honeybee ORNs, tetramethrin-induced tail currents decayed with a faster time course than permethrin-induced tail current, which would be in agreement with these earlier results since tetramethrin requires less pulses to reach a steady tail current level.

In summary, our work describes for the first time pyrethroid-induced sodium current modifications in voltage-clamped olfactory receptor neurons. Our results suggest that pyrethroids are as potent at modifying honeybee sodium channels as those from other insects. In addition to a traditionally reported tail current summation, we describe for the first time a pyrethroid-induced peak current increase that might contribute to the use-dependent mode of action of pyrethroids.

\section{Conflict of interest statement}

None

\section{Acknowledgments}

Authors wish to thank I. Bornard for scanning electron microscopy observations and the laboratory beekeepers J. Aptel, and J.P. Vermandère for maintaining an experimental apiary in Avignon and providing bees. We thank S. Diochot for her generous gift of the sea anemone toxin. We thank two anonymous referees for valuable comments on our manuscript and Cynthia McDonnell for English editing. The authors thank Région Provence-Alpes-Côte d'Azur and INRA-SPE scientific department for their financial support to AK (Ph.D fellowship). 


\section{References}

Barnes, S. \& Hille, B. (1988) Veratridine modifies open sodium channels. J Gen Physiol, 91, 421-443.

Blair, N.T. \& Bean, B.P. (2003) Role of tetrodotoxin-resistant Na+ current slow inactivation in adaptation of action potential firing in small-diameter dorsal root ganglion neurons. J Neurosci, 23, 10338-10350.

Bloomquist, J.R. (1996) Ion channels as targets for insecticides. Annu Rev Entomol, 41, 163190.

Chauzat, M.P. \& Faucon, J.P. (2007) Pesticide residues in beeswax samples collected from honey bee colonies (Apis mellifera L.) in France. Pest Management Science, 63, 11001106.

Choi, J.S. \& Soderlund, D.M. (2006) Structure-activity relationships for the action of 11 pyrethroid insecticides on rat $\mathrm{Na} v 1.8$ sodium channels expressed in Xenopus oocytes. Toxicol Appl Pharmacol, 211, 233-244.

Collet, C. (2009) Excitation-contraction coupling in skeletal muscle fibers from adult domestic honeybee. Pflug Arch Eur J Phy, 458, 601-612.

Collet, C. \& Belzunces, L. (2007) Excitable properties of adult skeletal muscle fibres from the honeybee Apis mellifera. J Exp Biol, 210, 454-464.

Cox, R.L. \& Wilson, W.T. (1984) Effects of Permethrin on the Behavior of Individually Tagged Honey Bees, Apis mellifera L. (Hymenoptera: Apidae). Environmental Entomology, 13, 375.

Decourtye, A., Devillers, J., Genecque, E., Le Menach, K., Budzinski, H., Cluzeau, S. \& Pham-Delegue, M.H. (2005) Comparative sublethal toxicity of nine pesticides on olfactory learning performances of the honeybee Apis mellifera. Arch Environ Contam Toxicol, 48, 242-250. 
Dong, K. (2007) Insect sodium channels and insecticide resistance. Invert Neurosci, 7, 17-30.

Du, Y., Nomura, Y., Liu, Z., Huang, Z.Y. \& Dong, K. (2009) Functional Expression of an Arachnid Sodium Channel Reveals Residues Responsible for Tetrodotoxin Resistance in Invertebrate Sodium Channels. Journal of Biological Chemistry, 284, 33869-33875.

Esslen, J. \& Kaissling, K.E. (1976) Number And Distribution Of Sensilla On Antennal Flagellum Of Honeybee (Apis-Mellifera-L). Zoomorphologie, 83, 227-251.

Feng, G., Deak, P., Chopra, M. \& Hall, L.M. (1995) Cloning and functional analysis of TipE, a novel membrane protein that enhances Drosophila para sodium channel function. Cell, 82, 1001-1011.

Gascuel, J., Masson, C., Bermudez, I. \& Beadle, D.J. (1994) Morphological analysis of honeybee antennal cells growing in primary cultures. Tissue Cell, 26, 551-558.

Ginsburg, K. \& Narahashi, T. (1999) Time course and temperature dependence of allethrin modulation of sodium channels in rat dorsal root ganglion cells. Brain Research, 847, 38-49.

Honeybee Genome Sequencing Consortium (2006) Insights into social insects from the genome of the honeybee Apis mellifera. Nature, 443, 931-949.

Jay, C. (1963) The development of honeybees in their cells. Journal of Apicultural Research, 2, 117-135.

Kadala, A., Charreton, M., Le Conte, Y., Jakob, I. \& Collet, C. (Year) Pyrethroids alter sodium channel gating in honeybee olfactory receptor neurons. European Chemoreception Research Organization XXth Congress. City.

Laurent, S., Masson, C. \& Jakob, I. (2002) Whole-cell recording from honeybee olfactory receptor neurons: ionic currents, membrane excitability and odourant response in developing workerbee and drone. Eur J Neurosci, 15, 1139-1152. 
Lee, D., Park, Y., Brown, T.M. \& Adams, M.E. (1999) Altered Properties of Neuronal Sodium Channels Associated with Genetic Resistance to Pyrethroids. Molecular Pharmacology, 55, 584-593.

Lund, A.E. \& Narahashi, T. (1981a) Kinetics of sodium channel modification by the insecticide tetramethrin in squid axon membranes. J Pharmacol Exp Ther, 219, 464473.

Lund, A.E. \& Narahashi, T. (1981b) Modification of sodium channel kinetics by the insecticide tetramethrin in crayfish giant axons. Neurotoxicology, 2, 213-229.

Lund, A.E. \& Narahashi, T. (1982) Dose-dependent interaction of the pyrethroid isomers with sodium channels of squid axon membranes. Neurotoxicology, 3, 11-24.

Martin, R.L., Pittendrigh, B., Liu, J., Reenan, R., ffrench-Constant, R. \& Hanck, D.A. (2000) Point mutations in domain III of a Drosophila neuronal Na channel confer resistance to allethrin. Insect Biochem Mol Biol, 30, 1051-1059.

Mullin, C.A., Frazier, M., Frazier, J.L., Ashcraft, S., Simonds, R., Vanengelsdorp, D. \& Pettis, J.S. (2010) High levels of miticides and agrochemicals in North American apiaries: implications for honey bee health. PLoS One, 5, e9754.

Narahashi, T. (1971) Mode of action of pyrethroids. Bull World Health Organ, 44, 337-345.

Park, Y., Lee, D., Taylor, M.F.J., Holloway, J.W., Ottea, J.A., Adams, M.E. \& Feyereisen, R. (2000) A Mutation Leu1029 to His in Heliothis virescens F. hscp Sodium Channel Gene Associated with a Nerve-Insensitivity Mechanism of Resistance to Pyrethroid Insecticides. Pesticide Biochemistry and Physiology, 66, 1-8.

Roy, M.L. \& Narahashi, T. (1992) Differential properties of tetrodotoxin-sensitive and tetrodotoxin-resistant sodium channels in rat dorsal root ganglion neurons. $J$ Neurosci, 12, 2104-2111. 
Smith, T.J., Ingles, P.J. \& Soderlund, D.M. (1998) Actions of the pyrethroid insecticides cismethrin and cypermethrin on house fly Vssc1 sodium channels expressed in Xenopus oocytes. Arch Insect Biochem Physiol, 38, 126-136.

Smith, T.J., Lee, S.H., Ingles, P.J., Knipple, D.C. \& Soderlund, D.M. (1997) The L1014F point mutation in the house fly Vssc1 sodium channel confers knockdown resistance to pyrethroids. Insect Biochem Mol Biol, 27, 807-812.

Soderlund, D.M. (2010) State-dependent modification of voltage-gated sodium channels by pyrethroids. Pesticide Biochemistry and Physiology, 97, 78.

Soderlund, D.M., Clark, J.M., Sheets, L.P., Mullin, L.S., Piccirillo, V.J., Sargent, D., Stevens, J.T. \& Weiner, M.L. (2002) Mechanisms of pyrethroid neurotoxicity: implications for cumulative risk assessment. Toxicology, 171, 3-59.

Song, J.-H., Nagata, K., Tatebayashi, H. \& Narahashi, T. (1996) Interactions of tetramethrin, fenvalerate and DDT at the sodium channel in rat dorsal root ganglion neurons. Brain Research, 708, 29-37.

Song, J.H. \& Narahashi, T. (1996) Modulation of sodium channels of rat cerebellar Purkinje neurons by the pyrethroid tetramethrin. J Pharmacol Exp Ther, 277, 445-453.

SupYoon, K., Symington, S.B., Hyeock Lee, S., Soderlund, D.M. \& Marshall Clark, J. (2008) Three mutations identified in the voltage-sensitive sodium channel [alpha]-subunit gene of permethrin-resistant human head lice reduce the permethrin sensitivity of house fly Vssc1 sodium channels expressed in Xenopus oocytes. Insect Biochemistry and Molecular Biology, 38, 296-306.

Tabarean, I.V. \& Narahashi, T. (1998) Potent Modulation of Tetrodotoxin-Sensitive and Tetrodotoxin-Resistant Sodium Channels by the Type II Pyrethroid Deltamethrin. $J$ Pharmacol Exp Ther, 284, 958-965. 
Tabarean, I.V. \& Narahashi, T. (2001) Kinetics of modulation of tetrodotoxin-sensitive and tetrodotoxin-resistant sodium channels by tetramethrin and deltamethrin. J Pharmacol Exp Ther, 299, 988-997.

Tan, J., Liu, Z., Nomura, Y., Goldin, A.L. \& Dong, K. (2002) Alternative splicing of an insect sodium channel gene generates pharmacologically distinct sodium channels. $J$ Neurosci, 22, 5300-5309.

Tatebayashi, H. \& Narahashi, T. (1994) Differential mechanism of action of the pyrethroid tetramethrin on tetrodotoxin-sensitive and tetrodotoxin-resistant sodium channels. $J$ Pharmacol Exp Ther, 270, 595-603.

Taylor, K.S., Waller, G.D. \& Crowder, L.A. (1987) Impairment of a classical conditioned response of the honey bee (Apis mellifera L.) by sublethal doses of synthetic pyrethroid insecticides. Apidologie, 18, 243-252.

Vais, H., Atkinson, S., Pluteanu, F., Goodson, S.J., Devonshire, A.L., Williamson, M.S. \& Usherwood, P.N.R. (2003) Mutations of the para Sodium Channel of Drosophila melanogaster Identify Putative Binding Sites for Pyrethroids. Mol Pharmacol, 64, 914-922.

Vais, H., Williamson, M.S., Goodson, S.J., Devonshire, A.L., Warmke, J.W., Usherwood, P.N. \& Cohen, C.J. (2000) Activation of Drosophila sodium channels promotes modification by deltamethrin. Reductions in affinity caused by knock-down resistance mutations. J Gen Physiol, 115, 305-318.

Warmke, J.W., Reenan, R.A., Wang, P., Qian, S., Arena, J.P., Wang, J., Wunderler, D., Liu, K., Kaczorowski, G.J., Van der Ploeg, L.H., Ganetzky, B. \& Cohen, C.J. (1997) Functional expression of Drosophila para sodium channels. Modulation by the membrane protein TipE and toxin pharmacology. J Gen Physiol, 110, 119-133.

Whitfield, C.W., Band, M.R., Bonaldo, M.F., Kumar, C.G., Liu, L., Pardinas, J.R., Robertson, H.M., Soares, M.B. \& Robinson, G.E. (2002) Annotated expressed sequence tags and 
cDNA microarrays for studies of brain and behavior in the honey bee. Genome Res, 12, 555-566.

Zhao, Y., Park, Y. \& Adams, M.E. (2000) Functional and evolutionary consequences of pyrethroid resistance mutations in S6 transmembrane segments of a voltage-gated sodium channel. Biochem Biophys Res Commun, 278, 516-521.

Zlotkin, E. (1999) The insect voltage-gated sodium channel as target of insecticides. Annu Rev Entomol, 44, 429-455. 

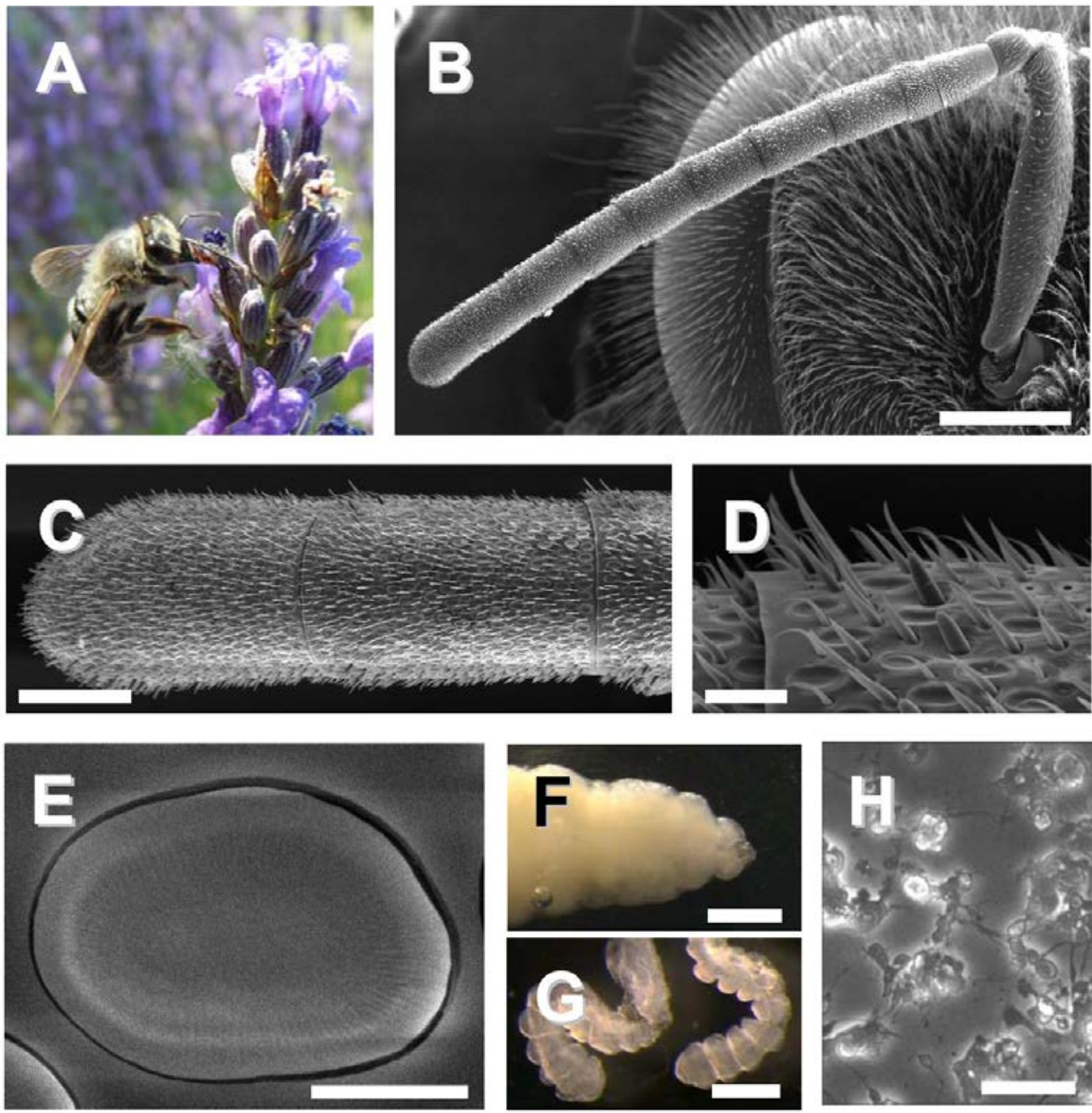

Fig. 1. Olfactory placodea sensilla from adult antennae and olfactory receptor neurons from prepupa in cell culture.

A, An adult honeybee foraging on lavender flowers. B, Scanning electron microscope image showing the antenna of a workerbee. The antenna is attached to the head through a joint and is composed of (from proximal to distal) the scalpa, the pedicel and the flagellum (made of 10 segments). C, Tip of the flagellum covered with a high density of sensillae at higher magnification. D, Several different kinds of sensillae are identified on the basis of their characteristic morphology (e.g. trichoid, placoid, basiconic, coeloconic). E, A single oval shaped placoid sensilla seen at higher magnification. F, Head of honeybee prepupae. G, Two prepupae antennae seen under a stereomicroscope. $\mathbf{H}$, Olfactory receptor neurons in culture after 2 days in vitro. Scale bars: B, $500 \mu \mathrm{m}$;

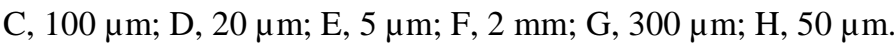



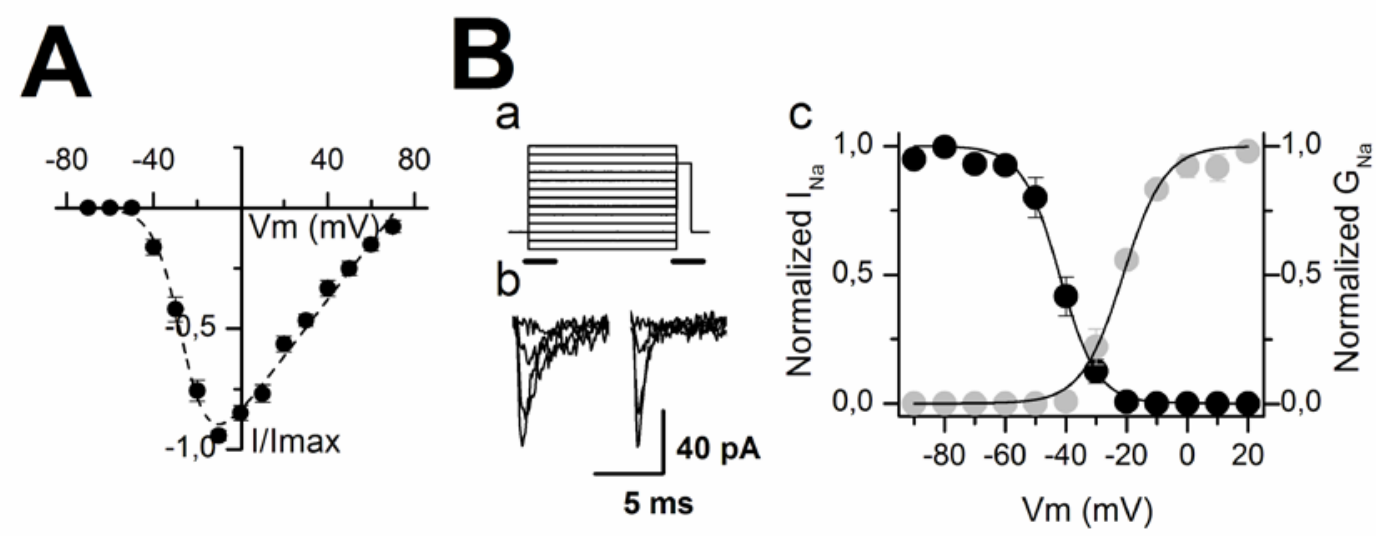

Fig. 2. Current-voltage relation and steady-state activation and inactivation curves for $\mathbf{I}_{\mathrm{Na}}$ from honeybee ORN.

A, Mean I/V curve obtained from twenty-two ORNs in response to depolarization pulses from a holding potential of $-80 \mathrm{mV}$. A theoretical curve was fitted to experimental data (dashed curve). B, A two-step pulse protocol (a) was used to measure simultaneously steady-state activation and inactivation properties. A $100 \mathrm{~ms}$ pulse (to $-100,-90, . .,+20 \mathrm{mV}$ ) was followed by a $10 \mathrm{~ms}$ pulse to $0 \mathrm{mV}$ from a holding potential of $-80 \mathrm{mV}$. $\mathrm{I}_{\mathrm{Na}}$ amplitude during these two successive pulses (example shown in $\mathrm{b}$ for depolarizations to $-60,-50,-40$ and -30 $\mathrm{mV}$ ), indicate the fraction of activated (grey circle) and inactivated (black circles) $\mathrm{I}_{\mathrm{Na}}$, respectively ( $\mathrm{n}=5$ neurons). Experimental points were fitted with two states Boltzman equations (solid curves, c). 
Figure 3


Fig. 3. Pyrethroid-induced sodium tail current in honeybee ORN.

A, In control conditions, no tail current could be detected upon repolarization from a 3 ms depolarizing pulse from -80 to $-10 \mathrm{mV}$, as a consequence of sodium channels fast deactivation properties (left trace). However, after three minutes superperfusion with a solution containing $10 \mu \mathrm{M}$ permethrin (a type I pyrethroid insecticide), a prominent tail current was observed upon repolarization to $-80 \mathrm{mV}$, essentially as a result of deactivation slowing down (middle trace). In the presence of tetrodotoxin $(1 \mu \mathrm{M})$, both the fast inactivating sodium current (activated during the pulse, $n=7)$ and the long lasting deactivation current $(n=3)$ were completely blocked (right trace). B, In the presence of permethrin $(10 \mu \mathrm{M})$, a two-step pulse protocol (upper traces) bringing membrane potential to $-80,-70, \ldots,+60 \mathrm{mV}$ right after the test pulse (to $-10 \mathrm{mV}$ ), was applied in order to explore the tail current reversal potential. Currents obtained from one ORN are presented below (for clarity, only traces obtained at $-80,-60,-40,-20,0,+20,+40$ and $+60 \mathrm{mV}$ are presented). C, Mean I/V curve for tail current amplitude in control (empty circles, $n=3$ ) and in the presence of permethrin $10 \mu \mathrm{M}$ (filled circles, $\mathrm{n}=2$ ). Apparent reversal potential of the tail current was estimated to be $+42 \mathrm{mV}$ by fitting a linear equation to experimental data. 


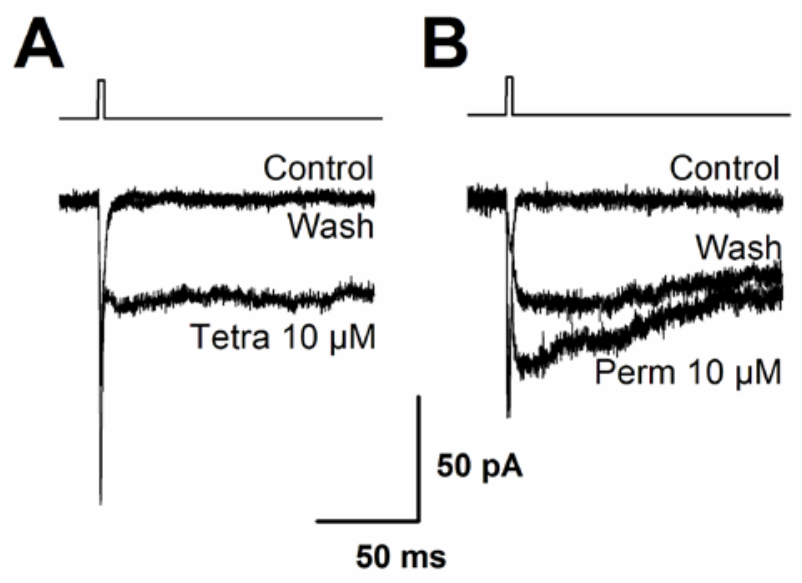

Figure 4

Fig. 4. Reversibility of tail current upon pyrethroid removal.

A, After exposure with tetramethrin $10 \mu \mathrm{M}$, a prominent and persistent tail current appears as compared with control conditions (same protocol as in Fig 3A). This current had completely disappeared 10 minutes after washout with a control solution. B, After exposure to permethrin $10 \mu \mathrm{M}$, a prominent and persistent tail current develops. This tail current persists even after 12 minutes washout with a control solution.

Figure 5


D



Fig. 5. Peak and late sodium current are differentially affected during exposure to both tetramethrin and permethrin.

After 3 minutes of exposure with tetramethrin $10 \mu \mathrm{M}$ (A) or permethrin $10 \mu \mathrm{M}(\mathbf{B})$, $\mathrm{I}_{\mathrm{Na}}$ peak (white circle) was not affected, whereas the $\mathrm{I}_{\mathrm{Na}}$ late current measured at the end of the $3 \mathrm{~ms}$ depolarization (black circle) was increased (same protocol as in Fig 3A). C, D, Mean values for experiments with tetramethrin (left panels) or permethrin (right panels) are presented for the peak (C) and the late current (D). For each panel, mean in control (left column) and pyrethroid-exposed conditions (right column) are represented. 
Figure 6


C


Fig. 6. Tail current decay and estimation of the fraction of modified sodium channels by tetramethrin and permethrin in ORNs.

After repolarization from a 3 ms pulse, the pyrethroid-induced tail current slowly returns towards zero. A, The decline of tail currents induced by tetramethrin $(10 \mu \mathrm{M})$ was estimated by measuring the residual tail current at the end of recordings, $130 \mathrm{~ms}$ after repolarization (arrow). B, On average, the tetramethrin-induced tail current reached $30 \%$ of its initial value (see text). C, The fraction of modified channels with tetramethrin was estimated in individual neurons according to Eqn 4 (see Methods). On average, $22 \pm 7 \%$ of the channels were modified by tetramethrin $10 \mu \mathrm{M}(\mathrm{n}=3$, see text). $\mathbf{D}$, In the presence of permethrin $10 \mu \mathrm{M}$, the residual tail current was measured $130 \mathrm{~ms}$ after the pulse (arrow) in eleven neurons. E, On average, the permethrin-induced tail current reached $51 \%$ of its initial value (see text). F, According to Eqn 4, permethrin $10 \mu \mathrm{M}$ modifies one fifth (20 $\pm 4 \%)$ of the channels on average ( $n=11$, see text). 
Figure 7

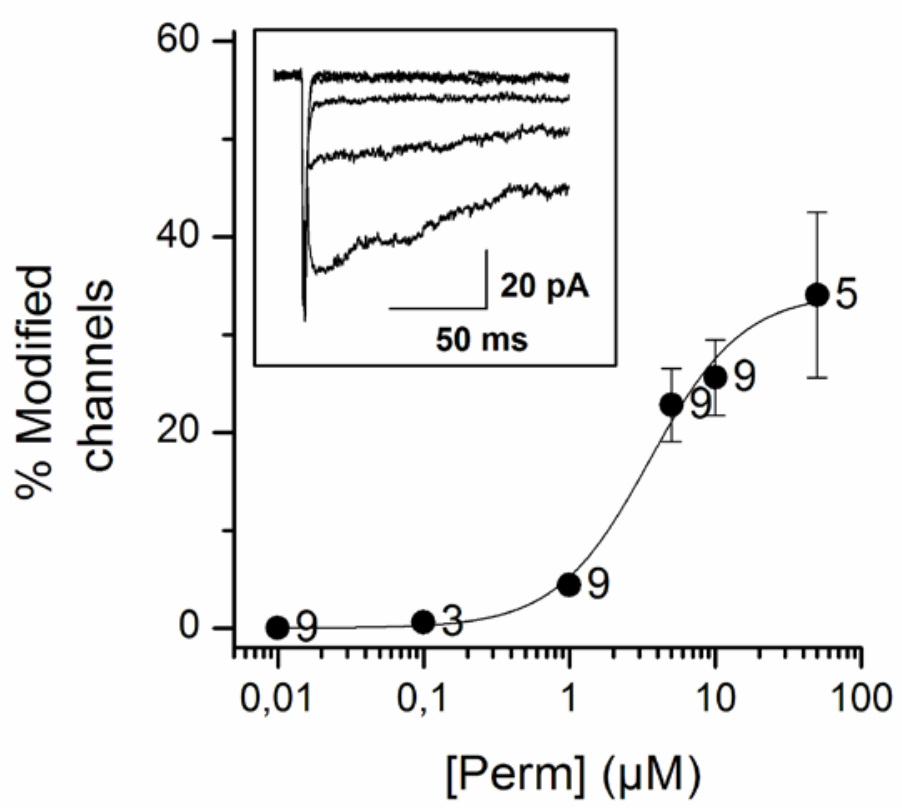

Fig.7. Percentage of sodium channels modified by various concentrations of permethrin.

In inset, superimposed tail currents obtained in one ORN in response to 3 ms-depolarization in control conditions and in the presence of permethrin $(0.1,1,5$ and $10 \mu \mathrm{M})$. Similar experiments were performed in 8 other ORNs with concentrations up to $50 \mu \mathrm{M}$. Average percentage of modified channels is given as a function of permethrin concentration (black circles), with the number of ORNs available for each concentration. Data were fitted by the Hill equation (see text). 




Fig. 8. Recovery from inactivation and use-dependent reduction of honeybee $\mathbf{I}_{\mathrm{Na}}$.

A, Paired-pulse protocols (inset, see text for details) were used to characterize kinetics of $\mathrm{I}_{\mathrm{Na}}$ recovery from inactivation. The $I_{N a}$ peak amplitude in response to the second pulse $\left(I_{2}\right)$ was normalized to $I_{N a}$ amplitude during the first pulse $\left(\mathrm{I}_{1}\right)$. In the first series of paired pulse experiments, interpulse intervals ( $\left.\Delta \mathrm{t}\right)$ were 1 to $13 \mathrm{~ms}$ with a $1 \mathrm{~ms}$ increment ( $\mathrm{n}=2$ neurons, each empty symbol type represent a single cell). In another series of paired pulse experiments, interpulse intervals $(\Delta \mathrm{t})$ were 3 to $23 \mathrm{~ms}$ with a $5 \mathrm{~ms}$ increment (n=6 neurons, each filled symbol type represents a single cell). Pulse duration was $3 \mathrm{~ms}$ and $5 \mathrm{~ms}$ in the first and the second protocol, respectively. Representative experimental traces obtained with these two kinds of protocols are shown in inset: upper and lower sets of traces obtained with $1 \mathrm{~ms}$ - and $3 \mathrm{~ms}$ - increment protocol, respectively. Recovery from inactivation follows an exponential time course. A single exponential with a time constant of 4.8 ms was fitted by eye to the data. B, Use-dependent reduction of $\mathrm{I}_{\mathrm{Na}}$ was assessed in individual neurons with $35 \mathrm{~Hz}$ (upper panel) and $125 \mathrm{~Hz}$ (middle panel) multipulse protocols (one depolarizing pulse every 28 and 8 ms, respectively). Lower panel shows the time course of the mean normalized $\mathrm{I}_{\mathrm{Na}}$ along with successive depolarization at $35 \mathrm{~Hz}$ $(\mathrm{n}=10)$ and $125 \mathrm{~Hz}(\mathrm{n}=5)$. 
A
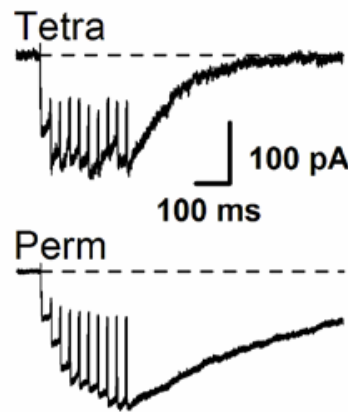

D

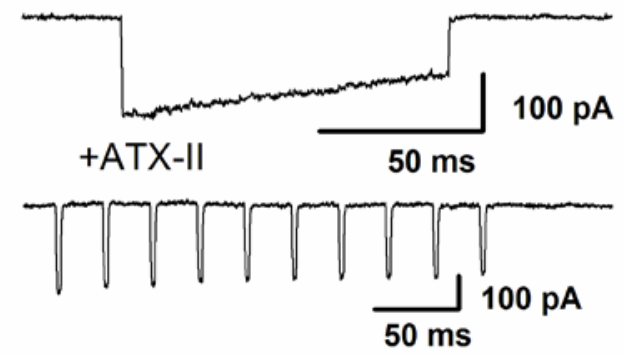

B

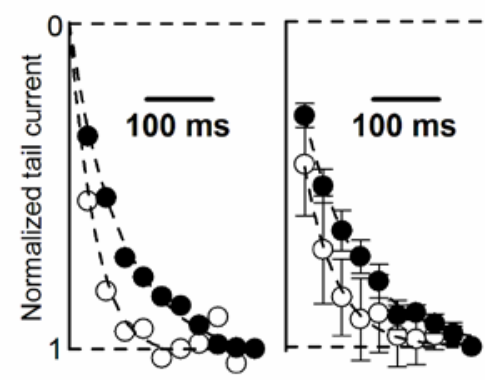

C



Fig. 9. Use-dependent accumulation of the pyrethroid-induced tail current.

A, Repetitive stimulations (ten 3 ms-pulses at $35 \mathrm{~Hz}$ ) lead to the progressive use-dependent development of a more sustained and larger pyrethroid-induced tail current. Tetramethrin appears more potent than permethrin at inducing this tail current accumulation, since the maximal effect (maximal tail current amplitude) is reached faster in the presence of tetramethrin. B, Left panel : the maximal tail current amplitude (normalized to the last pulse amplitude, to ease comparison) plotted as a function of time, from traces shown in $A$ (white and black dots for tetramethrin and permethrin, respectively, superimposed curves drawn by eye). Right panel : Averaged normalized tail currents for tetramethrin-exposed (white dots) and permethrin-exposed (black dots) neurons ( $\mathrm{n}=4$ and 13 ORNs, respectively). Superimposed monoexponential curves were fitted to the mean data obtained from tetramethrin and permethrin-exposed ORNs. C, In a majority of pyrethroid-treated neurons, the use-dependent tail current summation was accompanied by an increase in the peak of the sodium current along with stimulations as illustrated in this neuron (lower trace, arrows), whereas the opposite phenomenon was observed (a peak current decrease) in the control solution (same neuron, top trace). D, At $20 \mathrm{nM}$, the sea anemone toxin ATX-II induces the appearance of a slowly-inactivating component of the honeybee sodium current (top trace). Deactivation is not affected as evidenced by the lack of any tail current either after a single long depolarization or a train of short 3ms depolarizations (35 Hz). E, ATX-II (20 nM) allows for a recruitment of a large quantity of pyrethroid-modified inactivated channels right away after a single short pulse (3 ms, right trace), whereas it take several stimulations $(3 \mathrm{~ms}, 35 \mathrm{~Hz})$ for the pyrethroid-induced-tail current to approach this amplitude in the absence of ATX-II (left trace). 